\title{
Multivariate Functional Outlier Detection
}

\author{
Mia Hubert · Peter Rousseeuw · Pieter Segaert
}

Received: date / Accepted: date

\begin{abstract}
Functional data are occurring more and more often in practice, and various statistical techniques have been developed to analyze them. In this paper we consider multivariate functional data, where for each curve and each time point a $p$-dimensional vector of measurements is observed. For functional data the study of outlier detection has started only recently, and was mostly limited to univariate curves $(p=1)$. In this paper we set up a taxonomy of functional outliers, and construct new numerical and graphical techniques for the detection of outliers in multivariate functional data, with univariate curves included as a special case. Our tools include statistical depth functions and distance measures derived from them. The methods we study are affine invariant in $p$-dimensional space, and do not assume elliptical or any other symmetry.
\end{abstract}

Keywords Depth · Diagnostic plot · Functional data · Graphical display · Heatmap · Robustness

\section{Introduction}

Functional data are occurring more and more often in practice, and various statistical techniques have been developed to analyze this type of data. Standard references on functional data include Ramsay and Silverman $(2002,2006)$ and Ferraty and Vieu (2006). A functional data set typically consists of $n$ curves observed on a set of time points $t_{1}, \ldots, t_{T}$. In this paper we consider multivariate functional data, where for each curve and each time point a $p$-dimensional vector of measurements is observed. Several examples of multivariate functional data can be found in the literature. Popular examples include the bivariate gait data set containing the simultaneous variation

M. Hubert, P. Rousseeuw, P. Segaert

Department of Mathematics, KU Leuven, Celestijnenlaan 200B, BE-3001 Heverlee, Belgium

Tel.: +32-16-322023

E-mail: Mia.Hubert@wis.kuleuven.be 
of the hip and knee angles of 39 children (Ramsay and Silverman, 2006). Berrendero et al (2011) analyzed daily temperature data measured at 3,9 and $12 \mathrm{~cm}$ below ground during 21 days. Another example is the multi-lead ECG data set analyzed by Pigoli and Sangalli (2012) for which $p=8$. Here, the data contain measurements of the human heart activity at 8 different places on the body.

Nowadays statisticians are well aware of the potential effects of outliers on data analysis, and there is an extensive literature on finding outliers and developing methods that are robust to their influence; see e.g. Rousseeuw and Leroy (1987) and Maronna et al (2006). But for functional data the study of outlier detection has started only recently, and was mostly limited to univariate curves, i.e. $p=1$. Febrero-Bande et al (2008) identified two reasons why outliers can be present in functional data. First, gross errors can be caused by errors in measurements and recording or typing mistakes, which should be identified and corrected if possible. Second, outliers can be correctly observed data curves that are suspicious or surprising in the sense that they do not follow the same pattern as that of the majority of the curves. Using recorded $\mathrm{NO}_{x}$ levels in Barcelona as a case study, Febrero-Bande et al (2008) proposed the following outlier detection procedure for univariate functional data (i.e. $p=1$ ), consisting of three main steps:

1. For each curve, calculate its functional depth (several versions exist);

2. delete observations with depth below a cutoff $C$;

3. go back to step 1 with the reduced sample, and repeat until no outliers are found.

Step 3 was added in the hope of avoiding masking effects. The cutoff value $C$ was obtained by a bootstrap procedure.

A different approach was taken by Hyndman and Shang (2010) who noted that the above method lacks sensitivity to curves whose pattern is different from the majority but whose values at each time point look inconspicuous. Hyndman and Shang (2010) considered the observed curves $\left(Y_{i}\left(t_{1}\right), \ldots, Y_{i}\left(t_{T}\right)\right)$ as multivariate observations in $T$ dimensions and applied a robust PCA method to them, keeping the first two principal components. Next they computed the bagplot (Rousseeuw et al, 1999) of these bivariate points, or their highest density regions (Hyndman, 1996). However, it is not guaranteed that outliers can be detected by looking at the first two principal scores.

To visualize univariate functional data and investigate their spread as well as possible outliers, Sun and Genton (2011) proposed the functional boxplot. This new boxplot is a generalization of the univariate boxplot using functional depth as a replacement for the traditional univariate ranks. It shows, as a function of time, the deepest curve, the $50 \%$ central region (i.e. the envelope of the $50 \%$ deepest curves), and the fence. The latter is obtained by inflating the central region by 1.5 times its range. In this approach a curve is considered outlying if it ventures outside the fence in at least one time point.

The aims of this paper are twofold. We first set up a taxonomy of functional outliers in order to be able to classify different types of outlying behavior. Secondly, we construct new tools for the detection of outliers in multivariate functional data, with univariate curves included as a special case. These tools will aid in handling and interpreting outlying curves. For instance, curves with local gross errors should not necessarily be flagged as completely outlying, as this would lead to a substantial 
loss of information. The proposed methods will be affine invariant in $p$-dimensional space, an important theoretical and practical benefit for multivariate curves. Also, we will not assume elliptical or any other symmetry.

In the next section we will discuss different types of functional outlyingness and set up a taxonomy. In Section 3 we discuss halfspace depth and extend its use to detect outliers in multivariate and functional data. Section 4 introduces the skew-adjusted projection depth and a new diagnostic plot. In Section 5 we apply our new techniques to real data sets, before presenting our conclusions.

\section{Functional Outliers}

\subsection{Taxonomy of functional outliers}

Functional observations can be deviating from the majority of the curves in various ways. First of all, we distinguish isolated outliers that exhibit outlying behavior during a very short time interval. For univariate curves this type of outlyingness reduces to one or more spikes or peaks. An illustration is given in Figure 1 which plots proton nuclear magnetic resonance (NMR) spectra of 40 different wine samples (Larsen et al, 2006). The full data set contains measurements in the spectral region from 6.00 to 0.50 , but for clarity we focus on the region between wavelengths 5.62 and 5.37 only, for which $T=397$ measurements are available for each curve. The red curve 37 has large peaks around wavelength 5.4 and can be considered an isolated outlier.

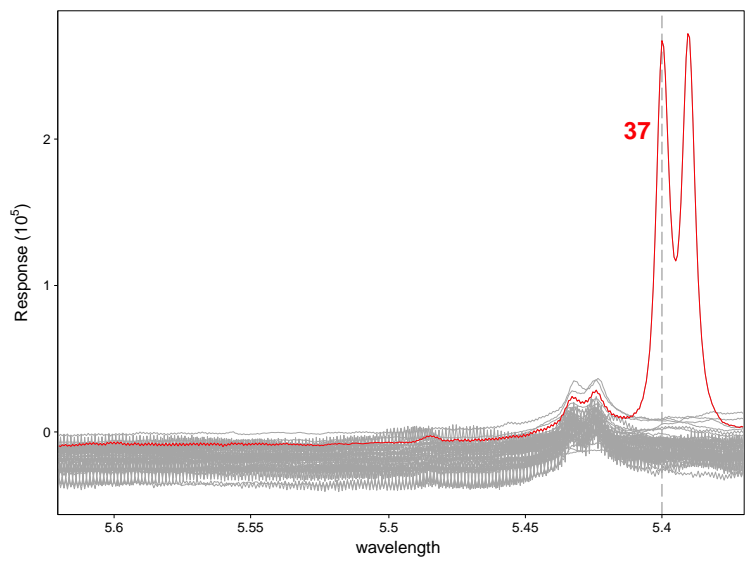

Fig. 1 NMR spectra for 40 wine samples with one isolated outlier.

The opposite of isolated outliers are persistent outliers, which we define as functional observations that are outlying on a large part of the domain, the most extreme case being the whole domain. Within this group we distinguish shift, amplitude and shape outliers. Shift outliers have the same shape as the majority, but are moved away. As an example we consider the tablets data set, depicted in Figure 2. This data set 
consists of near infrared spectroscopy responses for a batch of pills, measured at wavenumbers between $7398 \mathrm{~cm}^{-1}$ and $10507 \mathrm{~cm}^{-1}$. (Note that wavenumbers are expressed in units of inverse length.) They have been analyzed in Dyrby et al (2002) and can be downloaded from http://www.models.kvl.dk/tablets. The full data set consists of tablets of different sizes and different amounts of the active ingredients. We select the 70 observations corresponding to tablets weighing $90 \mathrm{mg}$ (shown in orange) and add 20 randomly selected observations of the tablets weighing $250 \mathrm{mg}$ (in purple). These two groups of tablets also differ in the amount of active components they contain. In the main group of the $90 \mathrm{mg}$ tablets, we note that the lowest curves are very similar to the others but display a downward shift, hence we can classify them as shift outliers. The $250 \mathrm{mg}$ tablets show an upward shift but they are also more wiggly around wavenumber 8224 . This difference becomes more pronounced when we consider the first derivatives of the curves in Figure 3. We now see that the blue curves have a different shape around wavenumber 8900 . Compared to the main group of the $90 \mathrm{mg}$ tablets we can consider the $250 \mathrm{mg}$ tablets as shape outliers, i.e. curves whose shape differs from the majority without necessarily standing out at any time point.

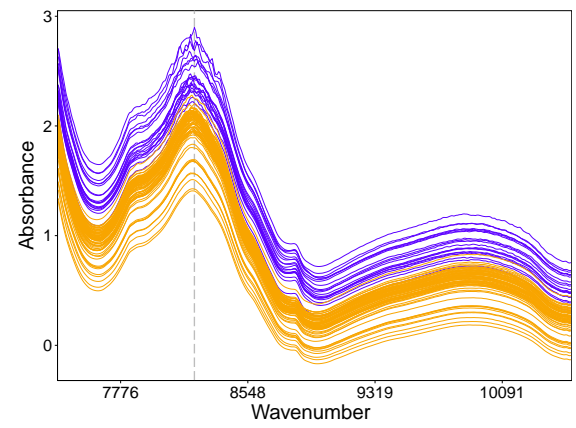

Fig. 2 The tablets data with the $90 \mathrm{mg}$ tablets colored orange and the $250 \mathrm{mg}$ tablets colored purple.

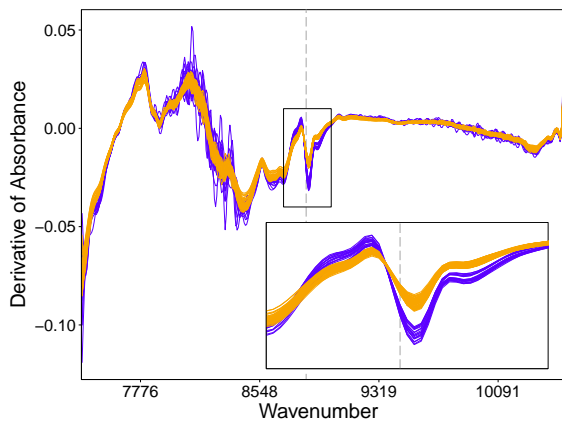

Fig. 3 Derivatives of the tablet curves, with the same color coding.

Another possibility is that a functional observation is outlying in an amplitude sense, i.e. the curve may have the same shape as the majority but its scale (range, amplitude) differs. This type of outlyingness is typically found in periodic processes. An example of an amplitude outlier will be discussed in Section 2.2.

Of course it will often happen that an abnormal curve exhibits a combination of outlying behavior. It can e.g. have several spikes in the first half of the domain, and a persistent outlying behavior in the second half. Or it can be shape outlying on one part of the domain and shift outlying on another.

Often functional data are preprocessed with a baseline correction, a warping procedure or other techniques, see for example Ramsay and Li (1998) and Wang and Gasser (1997). As a result it might happen that an outlying curve is transformed into a regular one. Baseline corrections will for example typically transform shift outliers into regular observations. In this case it can be useful to apply outlier detection tech- 
niques to the curves defining the transformation (such as the warping functions) or to include these curves in the analysis. This will be illustrated on the tablets data in Section 5.

\subsection{Functional outlier detection}

All examples shown so far are univariate functional data, for which outliers can often be detected just by looking at the curves. Automatic detection of isolated and shift outliers can then easily be carried out with standard outlier detection methods for univariate data applied to the measurements at each time point. For shape and amplitude outliers more advanced techniques are needed, such as those mentioned in Section 1. For multivariate functional data the problem is much harder. None of the outliers defined in Section 2.1 will necessarily be outlying in one of the marginals, hence it is not sufficient to analyze the components separately. An example is presented in LópezPintado et al (2014) where height and weight curves for boys separately do not show any marginal outlier, but a bivariate analysis flags one unusual infant. This illustrates that the correlation structure between the components should not be ignored. See also Hubert et al (2012) for other examples.

We illustrate this issue on the bivariate writing data which is a part of the Character Trajectories data set included in the UCI machine learning repository (Bache and Lichman, 2013). The data measure the horizontal and vertical coordinates of a pen tip while a person writes the letter 'i' (without the dot) $n=174$ times. The observed data were first interpolated to obtain $T=100$ equally spaced time points. The resulting bivariate functional observations are displayed in Figure 4 for the horizontal coordinates and in Figure 5 for the vertical ones. When we plot both coordinates as in Figure 6 the character ' $i$ ' is clearly visible, but this representation does not show the speed of writing. We highlighted three outlying curves with different behavior on all three figures. The dot-dashed red curve (case 67) roughly has the same shape as the regular curves, but it has a smaller scale. This curve can be classified as an amplitude outlier. The dashed blue observation (curve 41) deviates from most of the trajectories during a long period, especially in the vertical component. This is an example of a shape outlier, which stands out in one component during a substantial time period. Yet another shape outlier is the solid black curve 132. This curve does not stand out in the marginals but it is a multivariate outlier during a long time period. To illustrate this, Figure 7 shows a bivariate scatterplot of the coordinates at $t=5$. On this plot we see that trajectory 132 deviates from the other curves.

To detect outliers in functional data and classify them according to our taxonomy we will propose several approaches. By analogy with the non-functional case, we will first estimate the central tendency of the curves using a method which is robust to outliers. To this end we will use the multivariate functional median based on depth. Then we will construct an appropriate distance to compute the deviation of the curves from this center. 


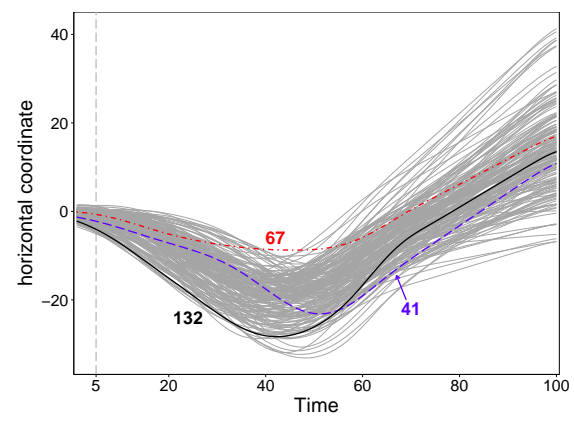

Fig. 4 Time profile of the horizontal coordinate of the writing data set.

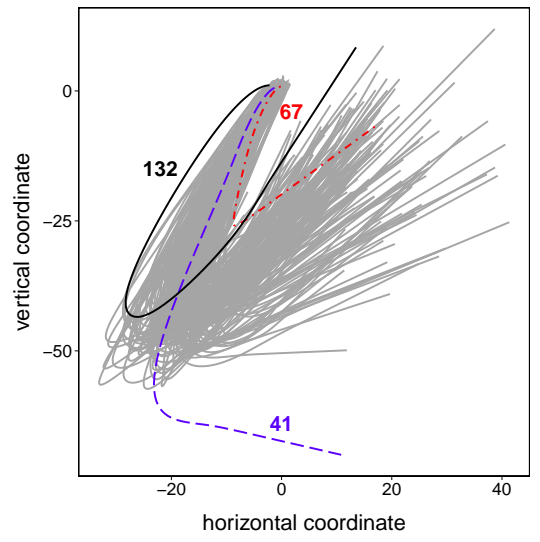

Fig. 6 Trajectories of the writing data set.

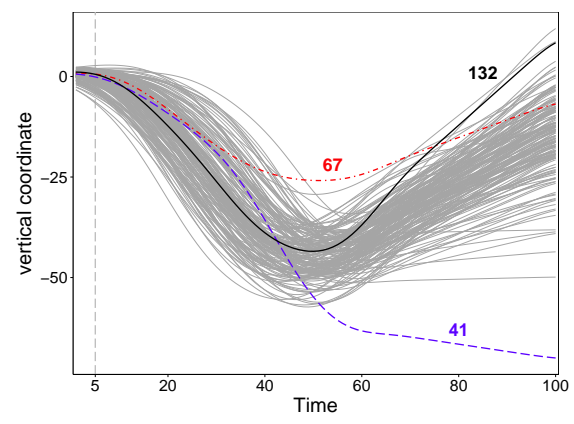

Fig. 5 Time profile of the vertical coordinate of the writing data set.

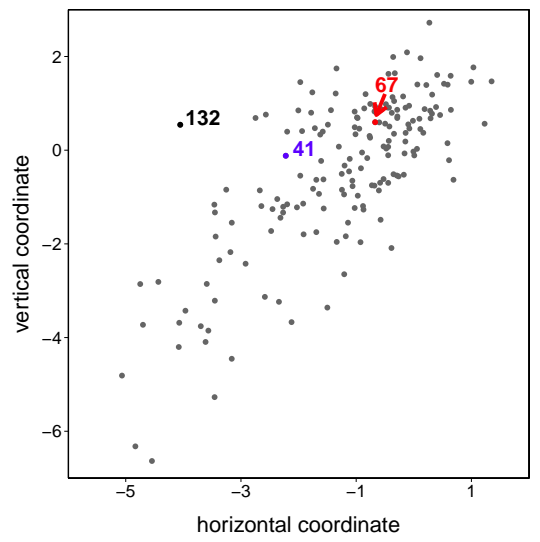

Fig. 7 Cross-sectional scatterplot of the writing data set at time point 5 .

\section{Outlier detection based on halfspace depth}

\subsection{Multivariate (functional) halfspace depth}

To measure the centrality of a point relative to a multivariate sample, Tukey (1977) introduced the halfspace depth. For simplicity of notation, we provide the definition in the population setting. Let $Y$ be a random variable on $\mathbb{R}^{p}$ with distribution $P_{Y}$, then the halfspace depth of any point $x \in \mathbb{R}^{p}$ relative to $P_{Y}$ is defined as the minimal probability mass contained in a closed halfspace with boundary through $x$ :

$$
\operatorname{HD}\left(x ; P_{Y}\right)=\inf _{\|v\|=1} P_{Y}\left\{v^{\prime} Y \geqslant v^{\prime} x\right\}
$$

Halfspace depth satisfies the requirements of a statistical depth function, as defined by Zuo and Serfling (2000a): it is affine invariant, attains its maximum value at the center of symmetry if there is one, is monotone decreasing along rays emanating from the center and vanishes at infinity. For any statistical depth function $D$ and for 
any $\alpha \in[0,1]$ the $\alpha$-depth region $D_{\alpha}$ contains the points whose depth is at least $\alpha$ :

$$
D_{\alpha}=\left\{x \in \mathbb{R}^{p} ; \mathrm{D}\left(x ; P_{Y}\right) \geqslant \alpha\right\} .
$$

The boundary of $D_{\alpha}$ is known as the $\alpha$-depth contour. The halfspace depth regions are closed, convex and nested for increasing $\alpha$. The halfspace median (or Tukey median) is defined as the center of gravity of the smallest non-empty depth region, i.e. the region containing the points with maximal halfspace depth. The finite-sample definitions of the halfspace depth, the Tukey median and the depth regions are obtained by replacing $P_{Y}$ by the empirical probability distribution $P_{n}$.

Properties of halfspace depth have been studied extensively. Donoho and Gasko (1992) derived many finite-sample properties, including the breakdown value of the Tukey median, whereas Romanazzi (2001) derived the influence function of the halfspace depth. Massé and Theodorescu (1994) and Rousseeuw and Ruts (1999) studied several properties of the depth and the depth contours at the population level. The asymptotic distribution of the Tukey median was given in Bai and He (1999), whereas He and Wang (1997) and Zuo and Serfling (2000c) studied the convergence of the depth regions and the contours. Continuity of the depth contours and the Tukey median was investigated in Mizera and Volauf (2002). Struyf and Rousseeuw (1999) showed that the halfspace depth function of a sample completely characterizes its empirical distribution, and this was later extended to the population case.

To compute the halfspace depth, several affine invariant algorithms have been developed. Exact algorithms in two and three dimensions and an approximate algorithm in higher dimensions have been proposed by Rousseeuw and Ruts (1996) and Rousseeuw and Struyf (1998). Recently Liu and Zuo (2014) proposed exact algorithms which are feasible up to $p=5$. Algorithms to compute the halfspace median have been developed by Rousseeuw and Ruts (1998) and Struyf and Rousseeuw (2000). To compute the depth contours the algorithm of Ruts and Rousseeuw (1996) can be used in the bivariate setting, whereas the algorithms developed in Hallin et al (2010) and Paindavaine and Šiman (2012) extend their applicability to at least $p=5$.

The bagplot (Rousseeuw et al, 1999) generalizes the univariate boxplot to bivariate data. As an example, Figure 8 shows the bagplot of the writing data at time $t=5$. The bag is the smallest $D_{\alpha}$ which contains $50 \%$ of the data points, and is darkcolored. The halfspace median is plotted as a red diamond. The fence, which itself is never drawn, is obtained by inflating the bag by a factor 3 relative to the median, and the data points outside of it are flagged as outliers and plotted as stars. The lightcolored loop is the convex hull of the points inside the fence. This bagplot has the nice property not to depend on any symmetry assumption: it works just as well for skewed data. That is, the bag need not be elliptically shaped and the median need not be positioned in the middle of the bag. In Figure 8 we see a moderate deviation from symmetry.

With the rise of functional data the notion of depth has been extended to this more complex situation. For univariate functional data the first proposals include the integrated depth of Fraiman and Muniz (2001), the $h$-modal depth of Cuevas et al (2006) and the random projection depth (Cuevas et al, 2007). The latter could also be applied to bivariate functional data. Later López-Pintado and Romo (2009, 2011) defined the (modified) band depth and the (modified) epigraph index. Recently the 


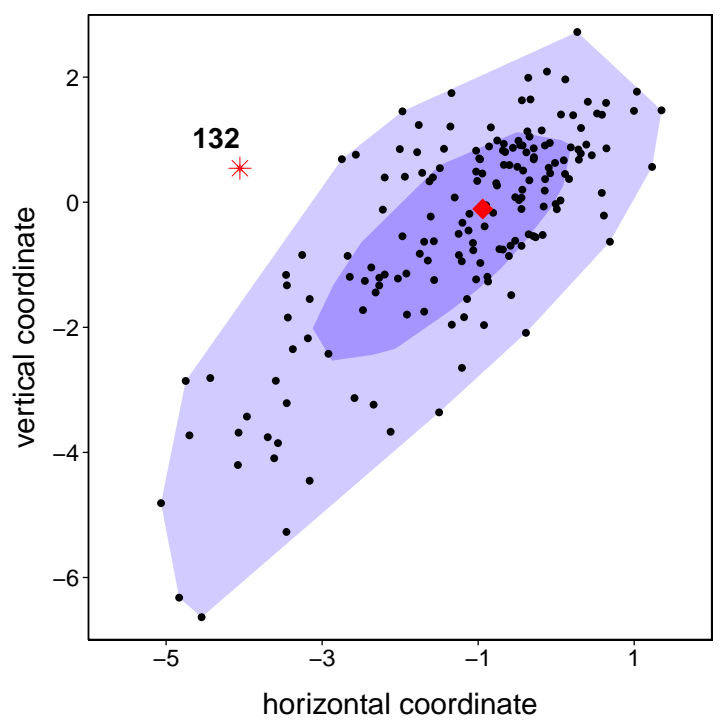

Fig. 8 Bagplot of the bivariate writing data at time $t=5$.

band depth was extended to multivariate functional data by Ieva and Paganoni (2013) and López-Pintado et al (2014). The latter approach can be seen as a particular case of the multivariate functional depth introduced and studied by Claeskens et al (2014).

We consider the observed curves as a realization of a $p$-variate stochastic process $\{Y(t), t \in U\}$ on $\mathbb{R}^{p}$ with $U=[a, b]$. We assume that it generates continuous paths in $C(U)^{p}$, the space of continuous functions from $U$ to $\mathbb{R}^{p}$. Following Claeskens et al (2014), with $D$ a statistical depth function on $\mathbb{R}^{p}$ and $w$ a function on $U$ which integrates to one, the Multivariate Functional Depth (MFD) of a curve $X \in C(U)^{p}$ with respect to the distribution $P_{Y}$ is defined as

$$
\operatorname{MFD}\left(X ; P_{Y}\right)=\int_{U} D\left(X(t) ; P_{Y}(t)\right) w(t) d t
$$

where $P_{Y}(t)$ is the distribution of $Y$ at time $t$. The MFD thus combines the local depths at each time point. By incorporating a weight function $w(t)$ one can emphasize or downweight certain time regions. Similarly to the multivariate setting, the functional median $\Theta(t)$ is defined as the curve with maximal MFD. The multivariate functional halfspace depth (MFHD) is obtained by taking the halfspace depth as the underlying depth function in (2). Properties of MFD, MFHD and the resulting medians have been studied by Claeskens et al (2014). In particular, if the multivariate depth $D$ has a unique maximum at each time point, say $\theta(t)$, then the MFD median $\Theta(t)$ equals $\theta(t)$ and the curve is continuous. 
For a functional data sample $\left(Y_{1}, \ldots, Y_{n}\right)$ measured at (not necessarily equidistant) time points $t_{1}, \ldots, t_{T}$ the finite-sample MFD of a curve $X$ is given by

$$
\operatorname{MFD}_{n}\left(X ; P_{n}\right)=\sum_{j=1}^{T} D\left(X\left(t_{j}\right) ; P_{n}\left(t_{j}\right)\right) W_{j}
$$

with $W_{j}=\int_{\left(t_{j-1}+t_{j}\right) / 2}^{\left(t_{j}+t_{j+1}\right) / 2} w(t) d t$ (and $\left.t_{0}=t_{1}, t_{T+1}=t_{T}\right)$ and $P_{n}\left(t_{j}\right)$ the empirical distribution of the observed curves at time $t_{j}$. For simplicity a uniform weight function will be used throughout this paper.

Since depth functions give an outward-center ordering, we expect that outliers will have a low depth value. The 'vanishing at infinity property' of a statistical depth function ensures that an observation will receive zero depth when it moves away from the center. The same holds for the MFD when the curve goes to infinity at almost all time points in the domain. Simulations in Claeskens et al (2014) have illustrated that several types of outliers, among which shifted outliers, indeed obtain a low MFHD depth. So it seems natural to use functional depth values for outlier detection. This was the underlying idea in Febrero-Bande et al (2008) where critical values were derived in a nonparametric way. A parametric approach was considered in Dang and Serfling (2010) but requires knowledge about the data distribution.

However, the depth values alone do not always provide sufficient information for outlier detection. First of all, not all observations with a low depth are indeed outlying. For example, observations on the boundary of the convex hull of a multivariate sample only have depth $1 / n$. In the functional setting the problem is even more challenging. In particular, isolated outliers will be very hard to detect using the MFD alone, as they will have a small multivariate depth value in only a small part of the domain. For instance, the hugely outlying curve 37 in Figure 1 has an MFHD value of 0.0973 while 5 of the 40 observations have a lower MFHD value.

Additional insight can be obtained by looking at the values of the depth at each time point. We illustrate this on the Octane data set consisting of 39 near infrared (NIR) spectra of gasoline samples (Esbensen, 2001; Rousseeuw et al, 2006). It is known that observations 25, 26 and 36-39 have a very different spectrum as they contain added ethanol, which is required in some states. Figure 9 clearly shows the six shape outliers, which are persistently outlying from wavelength 1390 onward.

When we order the spectra according to their MFHD, the six outliers have ranks $16,3,12,10,5$ and 15 respectively, hence they do not have the lowest functional depth. On the other hand spectrum 34 has the smallest depth since most of the time it lies near the lower edge of the regular data. Let us also look at the cross-sectional depth values by constructing a heatmap with $n$ rows and $T$ columns. Each cell of this map is colored according to $\operatorname{HD}\left(Y_{i}\left(t_{j}\right)\right)$ for $i=1, \ldots, n$ and $j=1, \ldots, T$, with the smallest depth value shown as white and the overall highest depth value colored dark green. On the vertical axis we rank the curves from top to bottom according to their MFHD value, thereby putting the deepest curve at the bottom. The color of the cell on the $i$ th row and the $j$ th column thus shows the halfspace depth at time $t_{j}$ of the curve with $i$ th smallest MFHD. This yields the halfspace depth heatmap in Figure 10. 


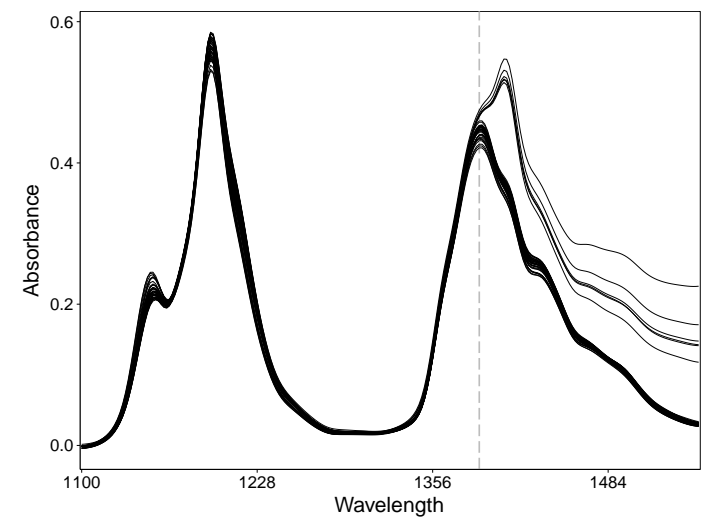

Fig. 9 Octane data set with 6 shape outliers.

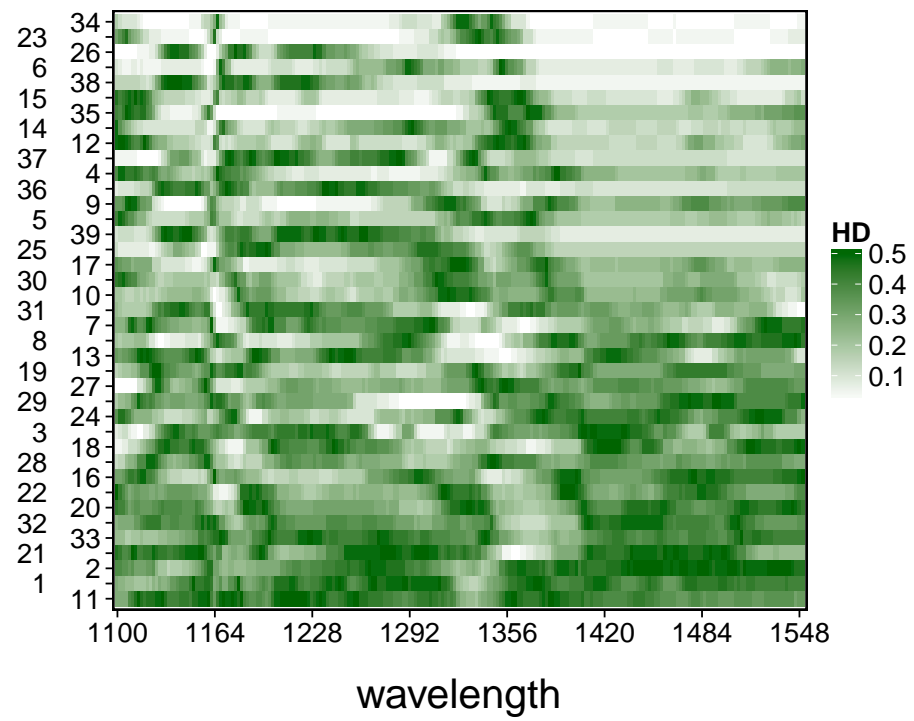

Fig. 10 Halfspace depth heatmap of the octane data.

Even with the HD heatmap it is still not easy to flag the outlying curves. We note several light regions in the heatmap, but they are scattered over many rows (curves) while some of the outliers (e.g. curve 25) even have a higher HD at the higher wavelengths than some of the regular curves. This is of course caused by the nature of halfspace depth which ranks the data from the outside inwards without considering their distance from the median. When the focus is on outlier detection, including a distance-based ranking is thus indispensable. This motivates the construction of a distance measure based on halfspace depth. 


\subsection{The bagdistance}

We first define a new statistical distance from a multivariate point $x \in \mathbb{R}^{p}$ to a random variable $Y$, based on the halfspace depth. This distance uses both the center and the dispersion of $Y$. As in the bagplot we define the bag $B$ as the $50 \%$ central region. This is the smallest depth region with at least $50 \%$ probability mass, i.e. $B=D_{\tilde{\alpha}}$ with $P_{Y}(B) \geqslant 0.5$, and $P_{Y}\left(D_{\alpha}\right)<0.5$ for all $\alpha>\tilde{\alpha}$. Next, we consider the ray from the halfspace median $\theta$ through $x$, and we define $c(x):=c_{x}$ as the intersection of this ray and the boundary of $B$. The bagdistance of $x$ to $Y$ is then given by the ratio between the Euclidean distance of $x$ to the halfspace median and the Euclidean distance of $c_{x}$ to the halfspace median:

$$
b d\left(x ; P_{Y}\right)=\frac{\|x-\theta\|}{\left\|c_{x}-\theta\right\|} .
$$

The denominator in (4) accounts for the dispersion of $Y$ in the direction of $x$. It is obvious that the bagdistance does not assume symmetry and is affine invariant.

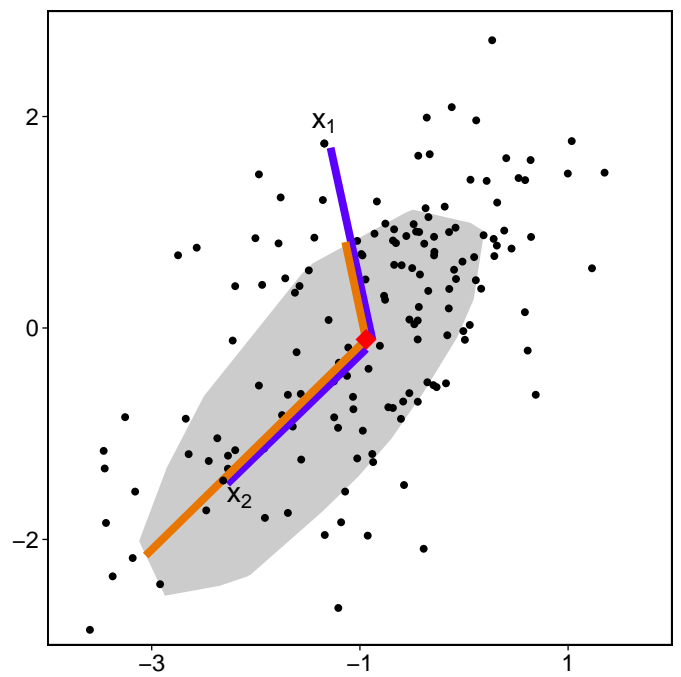

Fig. 11 Illustration of the bagdistance between an arbitrary point and a multivariate sample.

The finite-sample definition is similar and is illustrated in Figure 11. The gray colored bag is the smallest depth region that covers half of the sample points. For two points $x_{1}$ and $x_{2}$ their Euclidean distance to the halfspace median (red diamond) is marked with a dark blue line, whereas the orange lines correspond to the denominator of (4) and reflect how these distances are scaled differently. Whereas the lengths of the blue lines are the same, the bagdistance of $x_{1}$ is 2.01 and that of $x_{2}$ is only 0.63 . These values reflect the position of the points relative to the sample, one lying somewhat away from the most central half of the data and the other lying well within the central 
part. Note that the bagdistance is implicitly used in the bagplot, as the fence consists of the points whose bagdistance is at most 3. Also note that the bagdistance is a very different notion from the halfspace-outlyingness $O_{H}\left(x ; P_{Y}\right)=1-2 \mathrm{HD}\left(x ; P_{Y}\right)$ defined by Dang and Serfling (2010). The $O_{H}$ contains exactly the same ranking information as HD and is not very helpful for outlier detection either, as noted by the authors.

To compute the bagdistance of a point $x$ with respect to a $p$-variate sample we can indeed compute the bag and then the intersection point $c_{x}$. In small dimensions computing the bag is feasible, and it is worth the effort if the bagdistance needs to be computed for many points. In higher dimensions computing the bag is hard, and then a simpler and faster algorithm is to search for the multivariate point $c^{*}$ on the ray from $\theta$ through $x$ such that

$$
\mathrm{HD}\left(c^{*} ; P_{n}\right)=\underset{i}{\operatorname{median}}\left\{\mathrm{HD}\left(x_{i} ; P_{n}\right)\right\} .
$$

Since HD is monotone decreasing on the ray this is fairly fast, e.g. by means of the bisection algorithm.

When applied to elliptically symmetric distributions, the bagdistance is proportional to the Mahalanobis distance:

Theorem 1 If $P_{Y}$ is an elliptically symmetric distribution with center $\mu$ and shape matrix $\Sigma$, i.e. $f_{Y}(y) \sim|\Sigma|^{-1 / 2} h\left((x-\mu)^{\prime} \Sigma^{-1}(x-\mu)\right)$ for some nonnegative function $h$, then $b d\left(x ; P_{Y}\right)=k \sqrt{(x-\mu)^{\prime} \Sigma^{-1}(x-\mu)}$ for some constant $k$.

Proof We first note that under elliptical symmetry the halfspace median equals $\mu$. Next it is well-known that the halfspace depth regions are ellipsoids $D_{\alpha}=\{x ;(x-$ $\left.\mu)^{\prime} \Sigma^{-1}(x-\mu) \leqslant k_{\alpha}\right\}$ for some constant $k_{\alpha}$ depending on $\alpha$, see e.g. Massé (2004), from which the result follows.

The bagdistance can be used to construct a metric on the space $\mathbb{R}^{p}$ in the following way. Assume that the halfspace median is contained in the interior of the bag, that is, there exists a ball of radius $\varepsilon>0$ with center $\theta$ which lies entirely inside the bag. Then define the function

$$
g: \mathbb{R}^{p} \rightarrow \mathbb{R}^{p}: x \mapsto g(x)= \begin{cases}0 & \text { if } x=\theta \\ (x-\theta) /\left\|c_{x}-\theta\right\| & \text { elsewhere }\end{cases}
$$

with $c_{x}$ as in (4). The function $g$ is well-defined since our assumption implies that $\left\|c_{x}-\theta\right\|>0$ for every $x \neq \theta$. Now define the bagdistance between two arbitrary points as

$$
b d\left(x_{1}, x_{2}\right)=\left\|g\left(x_{1}\right)-g\left(x_{2}\right)\right\| .
$$

Theorem 2 If the halfspace median $\theta$ of a distribution $P$ lies in the interior of the bag, the bagdistance (5) is a metric on $\mathbb{R}^{p}$.

Proof It is clear that $b d\left(x_{1}, x_{2}\right)$ is well-defined and symmetric in its arguments. The triangle inequality follows readily from the triangle inequality for the Euclidean distance. Finally we note that $b d\left(x_{1}, x_{2}\right)=0$ implies $g\left(x_{1}\right)=g\left(x_{2}\right)$. Since the multivariate points $g\left(x_{1}\right)$ and $g\left(x_{2}\right)$ have the same direction it follows that $c_{x_{1}}=c_{x_{2}}$ implying $x_{1}=x_{2}$. 
This result continues to hold in the finite-sample case. We can also extend the bagdistance to the functional setting. The functional bagdistance of a curve to (the distribution of) a stochastic process $Y$ is defined as

$$
f b d\left(X ; P_{Y}\right)=\int_{U} b d\left(X(t) ; P_{Y}(t)\right) d t
$$

Note that in general it is possible that this distance becomes infinite.

As in the multivariate setting, the functional bagdistance can be extended to a distance between two arbitrary continuous functions $X_{1}$ and $X_{2}$ using the formula

$$
f b d\left(X_{1}, X_{2}\right)=\int_{U} b d\left(X_{1}(t), X_{2}(t)\right) d t .
$$

Under some weak conditions this is a metric:

Theorem 3 Assume $Y$ generates continuous paths in $C(U)^{p}$ such that

$$
m:=\inf _{t \in U} \inf _{x \in \mathbb{R}^{p}}\left\|c_{x}-\Theta(t)\right\|>0
$$

with $\Theta(t)$ the MFHD median. Then the functional bagdistance (7) is a metric on $C(U)^{p}$.

Proof Condition (8) implies that the bagdistance is defined for every $t \in U$. Without loss of generality we assume that $\Theta(t)=0$. Since $X_{1}, X_{2} \in C(U)^{p}$ and $U$ is compact, it follows for all $t \in U$ that $\left\|X_{1}(t)-X_{2}(t)\right\| \leqslant\left\|X_{1}(t)\right\|+\left\|X_{2}(t)\right\| \leqslant M$ for some $M<\infty$. Therefore

$$
\begin{aligned}
f b d\left(X_{1}, X_{2}\right) & =\int_{U} b d\left(X_{1}(t), X_{2}(t)\right) d t=\int_{U} d_{E}\left(\frac{X_{1}(t)}{\left\|c_{X_{1}(t)}\right\|}, \frac{X_{2}(t)}{\left\|c_{X_{2}(t)}\right\|}\right) d t \\
& \leqslant \int_{U} \frac{\left\|X_{1}(t)\right\|}{m}+\frac{\left\|X_{2}(t)\right\|}{m} d t \leqslant 2 \int_{U} \frac{M}{m} d t .
\end{aligned}
$$

Since $U$ is compact the latter integral is finite. Clearly $f b d$ is symmetric and the triangle inequality holds. Finally $f b d\left(X_{1}, X_{2}\right)=0$ implies that $b d\left(X_{1}(t), X_{2}(t)\right)=0$ for almost every time point since $b d$ is positive. This in turn implies that $X_{1}(t)=X_{2}(t)$ at almost every time point, and since they are continuous, $X_{1}=X_{2}$.

The multivariate and functional bagdistance now allow us to construct a diagnostic plot which shows the global outlyingness of the curve as well as the local outlyingness at each time point. We again construct a heatmap, where now we color the cells of each row according to the cross-sectional bagdistance of the corresponding curve. The rows are sorted in decreasing order of their functional bagdistance, so that the outliers should typically be on top (as in the depth heatmap). The color of the cell on the $i$ th row and the $j$ th column thus corresponds to the bagdistance at time $t_{j}$ of the curve with $i$ th largest functional bagdistance. To emphasize that this plot focuses on the outlyingness of curves we use red tints. The depth heatmap of Section 3.1 used green tints instead, as it shows the degree of centrality.

The bagdistance heatmap of the octane data set in Figure 12 now clearly flags the six outliers. They have the highest functional bagdistance, and they become outlying from the same time point onward. 


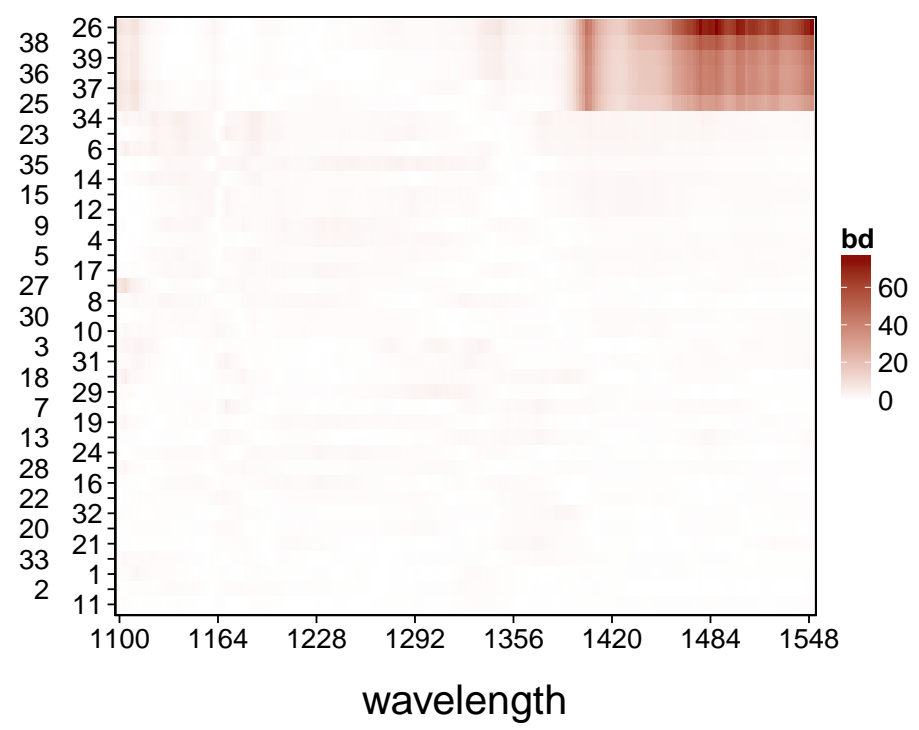

Fig. 12 Bagdistance heatmap of the octane data set.

\section{Outlier detection based on adjusted outlyingness}

\subsection{Skew-adjusted projection depth}

As an alternative to the HD based heatmaps, we also construct diagnostic plots based on a different depth function. Since the introduction of halfspace depth various other affine invariant depth functions have been defined, among which simplicial depth (Liu, 1990) and projection depth (Zuo, 2003) which is essentially the inverse of the Stahel-Donoho outlyingness (SDO). An overview of depth functions can be found in Mosler (2013). The SDO is based on the geometrical insight that a multivariate outlier should be outlying in at least one direction. The idea is to project the data onto many lines and to use a robust univariate measure of outlyingness on the projections (Stahel, 1981; Donoho, 1982). The population SDO of an arbitrary point $x$ with respect to a random variable $Y$ with distribution $P_{Y}$ is defined as

$$
\operatorname{SDO}\left(x ; P_{Y}\right)=\inf _{\|v\|=1} \frac{\left|v^{\prime} x-\operatorname{median}\left(v^{\prime} Y\right)\right|}{\operatorname{MAD}\left(v^{\prime} Y\right)}
$$

from which the projection depth is derived:

$$
\mathrm{PD}\left(x ; P_{Y}\right)=\frac{1}{1+\operatorname{SDO}\left(x ; P_{Y}\right)} .
$$

Since the SDO has an absolute deviation in the numerator and uses the MAD in its denominator it is best suited for symmetric distributions. For asymmetric distributions Brys et al (2005) proposed the adjusted outlyingness (AO) in the context of 
robust independent component analysis. The AO uses the medcouple (MC) of (Brys et al, 2004) as a robust measure of skewness. The medcouple of a univariate dataset $\left\{z_{1}, \ldots, z_{n}\right\}$ is defined as

$$
\operatorname{MC}\left(z_{1}, \ldots, z_{n}\right)=\underset{i, j}{\operatorname{median}} \frac{\left(z_{j}-\operatorname{median}_{k} z_{k}\right)-\left(z_{i}-\operatorname{median}_{k} z_{k}\right)}{z_{j}-z_{i}}
$$

where $i$ and $j$ have to satisfy $z_{i} \leqslant \operatorname{median}_{k}\left(z_{k}\right) \leqslant z_{j}$ and $z_{i} \neq z_{j}$. Note that $-1<\mathrm{MC}<$ 1 and $\mathrm{MC}=0$ for symmetric distributions, whereas $\mathrm{MC}>0$ indicates right skewness and $\mathrm{MC}<0$ indicates left skewness.

The adjusted outlyingness $\mathrm{AO}$ is defined as:

$$
\operatorname{AO}\left(x ; P_{Y}\right)=\sup _{\|v\|=1} \begin{cases}\frac{v^{\prime} x-\operatorname{median}\left(v^{\prime} Y\right)}{w_{2}\left(v^{\prime} Y\right)-\operatorname{median}\left(v^{\prime} Y\right)} & \text { if } v^{\prime} x>\operatorname{median}\left(v^{\prime} Y\right) \\ \frac{\operatorname{median}\left(v^{\prime} Y\right)-v^{\prime} x}{\operatorname{median}\left(v^{\prime} Y\right)-w_{1}\left(v^{\prime} Y\right)} & \text { if } v^{\prime} x<\operatorname{median}\left(v^{\prime} Y\right)\end{cases}
$$

where

$$
\begin{aligned}
& w_{1}(Z)=Q_{1}(Z)-1.5 e^{-4 \mathrm{MC}(Z)} \mathrm{IQR}(Z) \\
& w_{2}(Z)=Q_{3}(Z)+1.5 e^{+3 \mathrm{MC}(Z)} \mathrm{IQR}(Z)
\end{aligned}
$$

if $\mathrm{MC}(Z) \geqslant 0$, where $Q_{1}(Z)$ and $Q_{3}(Z)$ denote the first and third quartile of a univariate random variable $Z$, and $\operatorname{IQR}(Z)=Q_{3}(Z)-Q_{1}(Z)$. If $\operatorname{MC}\left(v^{\prime} Y\right)<0$, we replace $v$ by $-v$. The denominator of (11) corresponds to the whiskers of the univariate adjusted boxplot proposed by Hubert and Vandervieren (2008). Other applications of the AO were studied by Hubert and Van der Veeken $(2008,2010)$.

We now define the skew-adjusted projection depth (SPD) as

$$
\operatorname{SPD}\left(x ; P_{Y}\right)=\frac{1}{1+\mathrm{AO}\left(x ; P_{Y}\right)} .
$$

Theorem 4 Let $\mathscr{P}$ be the set of all probability distribution functions on $\mathbb{R}^{p}$. The mapping $D(\cdot, \cdot): \mathbb{R}^{p} \times \mathscr{P} \rightarrow \mathbb{R}:(x, P) \mapsto S P D(x, P)$ is a statistical depth function according to Definition 2.1 of Zuo and Serfling (2000a) and its depth regions are convex.

Proof It is clear that $D(\cdot, \cdot)$ is bounded and non-negative. Since AO is affine invariant, the skew-adjusted projection depth will be so as well. It is further evident that SPD vanishes at infinity. Note that being an $H$-symmetric distribution about a unique point $\theta \in \mathbb{R}^{p}$ is equivalent to saying that median $\left(v^{\prime} Y\right)=v^{\prime} \boldsymbol{\theta}$ for any unit vector $v \in \mathbb{R}^{p}$ (Zuo and Serfling, 2000b). Maximality at the center and monotonicity relative to the deepest point follow immediately.

The convexity of the depth regions follows from the convexity of the adjusted outlyingness function $\mathrm{AO}(\cdot ; P)$. For univariate data, the $\mathrm{AO}$ is a piecewise linear function composed of a downward sloping line ending in the point $(\operatorname{median}(P), 0)$ and an upward sloping line starting in the same point, so it is convex. For multivariate data, the AO relative to a single direction $v$ is thus convex too. Therefore the multivariate AO is a supremum (over all $v$ ) of convex functions. 
To compute the finite-sample SPD we have to rely on approximate algorithms as it is infeasible to consider all directions $v$. A convenient affine invariant procedure is obtained by considering directions $v$ which are orthogonal to an affine hyperplane through $p+1$ randomly drawn data points. In our implementation we use $250 p$ directions.

Since the SPD is a bona fide depth function, we can plug it into (2) yielding the multivariate functional skew-adjusted projection depth (MFSPD). The MFSPD therefore satisfies the properties in Claeskens et al (2014). In particular, the resulting median is continuous when at each time point the multivariate depth $D$ has a unique maximum.

On the other hand we can also integrate the AO itself, yielding the functional adjusted outlyingness

$$
\operatorname{FAO}\left(x ; P_{Y}\right)=\int_{U} \mathrm{AO}\left(X(t) ; P_{Y}(t)\right) d t
$$

Throughout this paper we will use the skewness-adjusted AO and SPD and their functional versions instead of the original SDO and PD, because the new tools can deal with symmetric as well as asymmetric data, and they have the same good properties. This is a kind of 'insurance policy' against skewness. As suggested by a referee, we could easily monitor the amount of skewness over time, for instance by plotting the largest absolute value of the medcouple encountered in the computation at each time point.

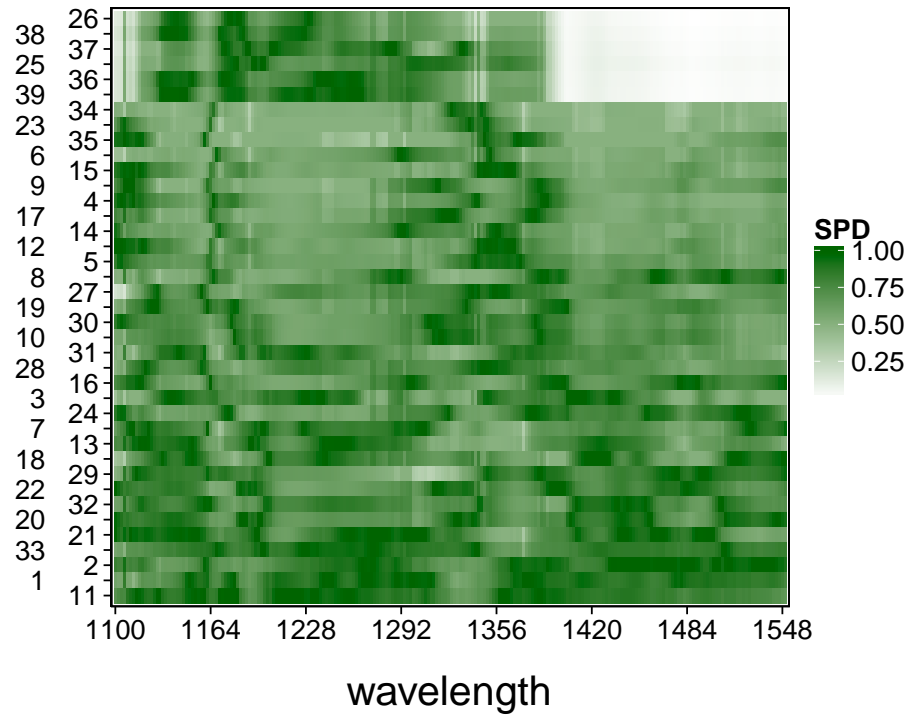

Fig. 13 Skew-adjusted projection depth (SPD) heatmap of the octane data. 
Similar to the heatmaps based on halfspace depth and the bagdistance we can construct the skew-adjusted projection depth heatmap and the adjusted outlyingness heatmap. The SPD heatmap of the octane data is shown in Figure 13. The six outliers are now clearly identifiable. In this example the outliers also have the lowest MFSPD, as they have a high $\mathrm{AO}$ over a rather long period.

The AO heatmap of the wine data is shown in Figure 14. Instead of showing all 40 rows of the heatmap, which would make the labels harder to read, we have zoomed in on the top 24 rows. Curve 37 jumps out immediately, as the two peaks we clearly saw in the raw data are dark colored. To make the details of the other curves more visible, we also display the same heatmap by setting the colors of curve 37 to white, and rescaling the colors of the other cells. The result is in Figure 15. We now also see the wiggly behavior of several curves.

Let us now take a closer look at the curves 35,23,3, and 2 which are at the top of the heatmap. Curves 35 and 23 show increased cross-sectional outlyingness around wavelengths 5.37, 5.43 and 5.50. At other wavelengths these spectra have almost constant outlyingness. In Figure 16 we see that they are mild shift outliers. Curves 2 and 3 have an even more stable adjusted outlyingness profile. They can be recognized as the bottom curves in Figure 16.

\subsection{Centrality-stability plot}

The relation (12) between SPD and AO will allow us to construct another diagnostic plot. We start by rewriting the $\mathrm{MFSPD}_{n}$ of a curve $X$ as

$$
\begin{aligned}
\operatorname{MFSPD}_{n}\left(X ; P_{n}\right) & =\sum_{j=1}^{T} \operatorname{SPD}_{n}\left(X\left(t_{j}\right) ; P_{n}\left(t_{j}\right)\right) W_{j} \\
& =\sum_{j=1}^{T} \frac{1}{\left(1+\mathrm{AO}\left(X\left(t_{j}\right) ; P_{n}\left(t_{j}\right)\right) W_{j}^{-1}\right.} \\
& =T\left[\underset{j}{\operatorname{hmean}\left(\left(1+\mathrm{AO}\left(X\left(t_{j}\right) ; P_{n}\left(t_{j}\right)\right) W_{j}^{-1}\right)\right]^{-1}}\right.
\end{aligned}
$$

where we use the abbreviation hmean $_{j}$ for the harmonic mean over $j=1, \ldots, T$. The MFSPD can thus be seen as the inverse of the harmonic mean of the weighted adjusted outlyingness in cross-sections. When we make a scatterplot of

$$
\left(\operatorname{MFSPD}_{n}\left(Y_{i} ; P_{n}\right) ; \underset{j}{\operatorname{ave}}\left(\left(1+\mathrm{AO}\left(Y_{i}\left(t_{j}\right) ; P_{n}\left(t_{j}\right)\right) W_{j}^{-1}\right)\right)\right.
$$

for all $i=1, \ldots, n$, the points are bounded from below by the function $z \rightarrow \frac{T}{z}$ since the arithmetic mean is always larger than or equal to the harmonic mean.

This scatterplot is shown in Figure 17 for the octane data and in Figure 18 for the wine data. The red curve is the theoretical lower bound. We see in both examples that the outliers stand out very clearly on the vertical axis. To understand this behavior, we reconsider the relation between the hmean and the arithmetic mean of a set of positive values. These means can only be equal if all values coincide. Therefore a 


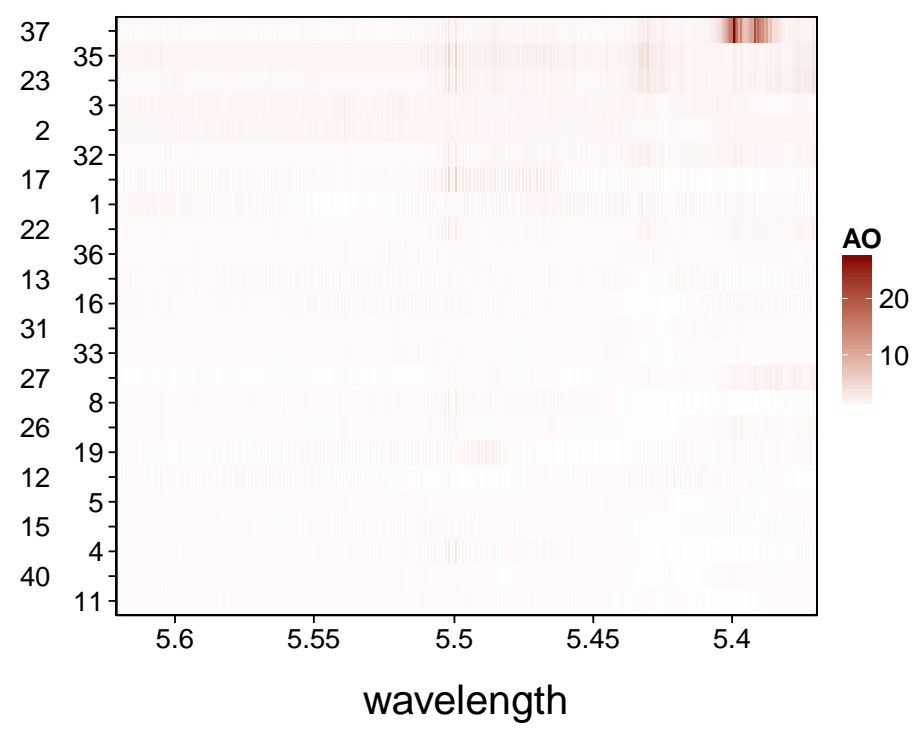

Fig. 14 Adjusted outlyingness (AO) heatmap of the wine data (top 24 rows).

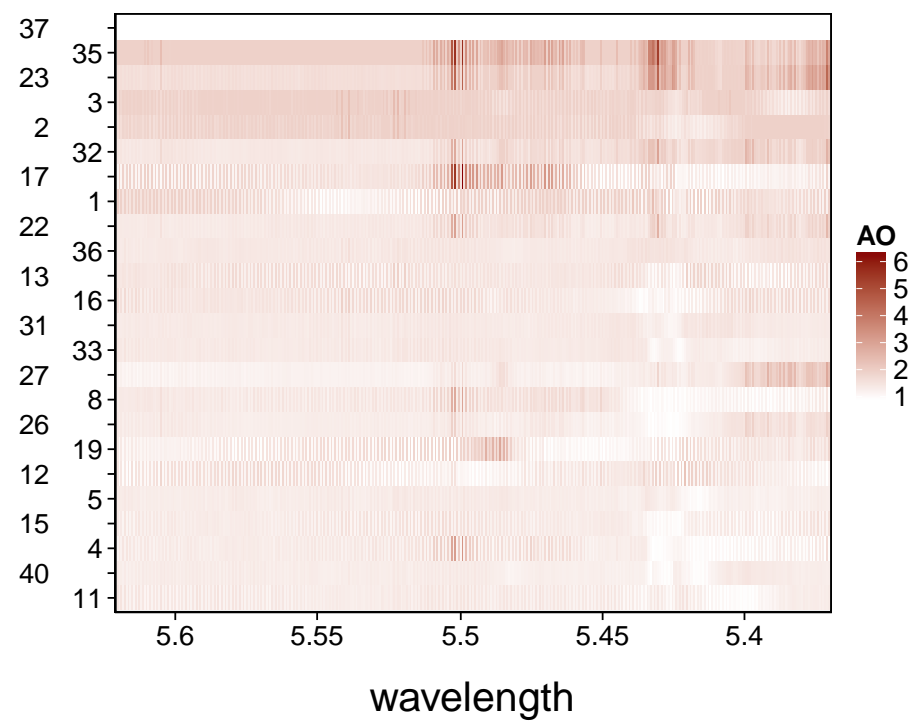

Fig. 15 Wine data: rescaled AO heatmap (by blanking out the top row). 


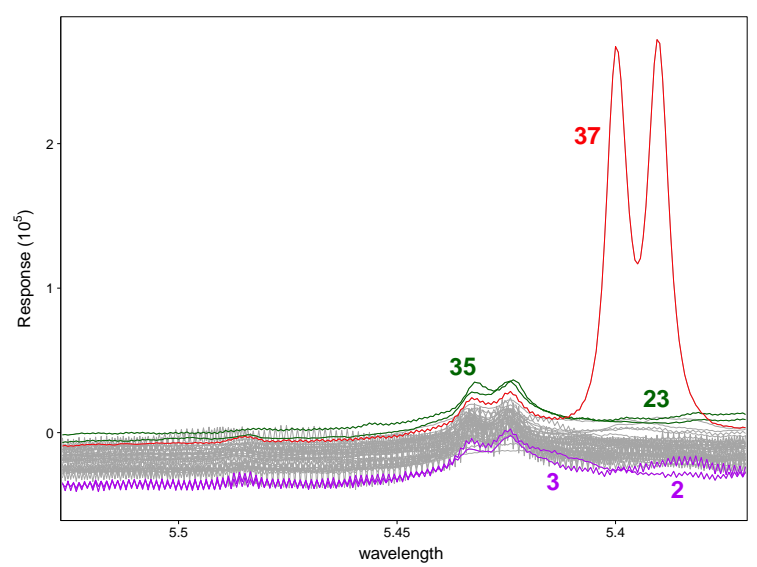

Fig. 16 Wine data with some curves showing outlying behavior.

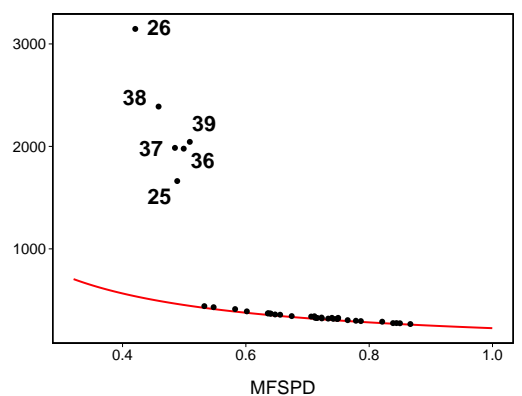

Fig. 17 Scatterplot of (14) for the octane data.

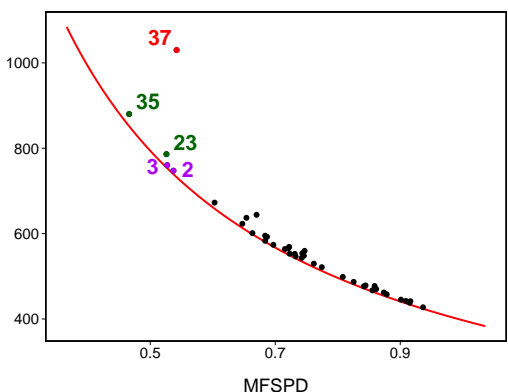

Fig. 18 Scatterplot of (14) for the wine data.

curve would be represented by a point on the lower bound if and only if its AO values would be constant over time. The vertical deviation from the lower bound thus reflects the variability of the degree of outlyingness over time.

Finally we construct the centrality-stability plot as the scatterplot of

$$
\left(1-\operatorname{MFSPD}_{n}\left(Y_{i} ; P_{n}\right) ; \underset{j}{\operatorname{ave}}\left(\left(1+\operatorname{AO}\left(Y_{i}\left(t_{j}\right) ; P_{n}\left(t_{j}\right)\right) W_{j}^{-1}\right)-\frac{T}{\operatorname{MFSPD}_{n}\left(Y_{i} ; P_{n}\right)}\right)\right.
$$

for all $i=1, \ldots, n$. The horizontal axis thus measures a curve's overall deviation from centrality, and the vertical axis its AO's deviation from stability. The deepest curves will lie in the left part of the plot, while the less central ones are to the right. Typically an isolated outlier will be in the upper region, as it is well-known that the arithmetic mean of positive numbers is more sensitive to outlying values than the harmonic mean. We expect shift outliers in the lower right region of this diagnostic plot (as they have a rather constant $\mathrm{AO}$ ) and shape/amplitude outliers in the upper right region.

Figure 19 shows the centrality-stability plot of the wine data. The isolated outlier 37 is again very visible. This curve is not very central either, as also outside of the two peaks it is in the outskirts of the curves. Curves 2, 3, 23 and 35 are at the 


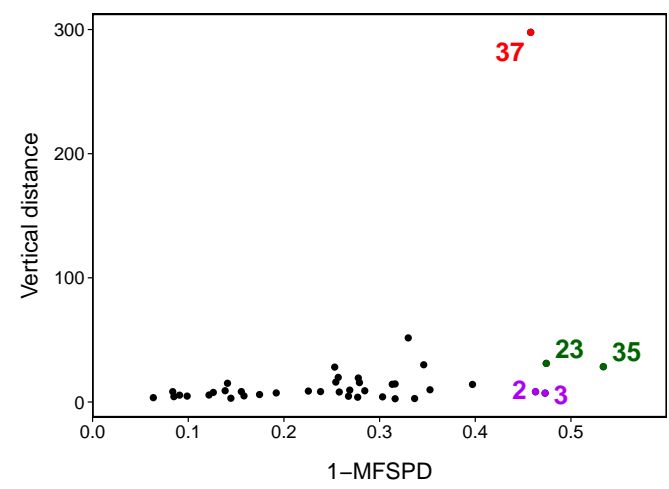

Fig. 19 Centrality-stability plot of the wine data.

bottom right of the plot. We already noted in Figure 16 that curves 2 and 3 have stable adjusted outlyingness profiles across wavelengths. This is confirmed by their low vertical coordinates in the centrality-stability plot.

\section{Real data analysis}

Whereas the examples in Sections 3 and 4 were univariate functional data sets, we now apply our new methodology to two multivariate data sets.

\subsection{Writing data}

The writing data were introduced in Subsection 2.2, where we also described the outlying behavior of some of the curves (41,67 and 132). The centrality-stability plot of this bivariate functional data set is shown in Figure 20, the trajectories are displayed in Figure 21 and their marginals in Figures 22 and 23. In the centrality-stability plot observations 41 and 132 indeed stand out. The MFSPD depth of the blue curve 41 is not extremely small (as seen on the horizontal axis of the centrality-stability plot), but its outlyingness is quite variable, resulting in a high vertical coordinate in Figure 20.

The solid black curve 132 has the lowest MFSPD although it does not look very outlying in the marginals, but it is outlying in the multivariate sense as we saw in Figures 7 and 8. From its vertical coordinate in the centrality-stability plot we conclude that the cross-sectional outlyingness does vary somewhat over time. The dot-dashed red curve 67 is in the outskirts of the data judging from its MFSPD on the horizontal axis in Figure 20, and its vertical position in that plot tells us that its $\mathrm{AO}$ values are quite variable. Curves 30 (dashed green), 95 (solid purple) and 121 (dot-dashed orange) are even higher in the centrality-stability plot, and we see that they are mostly outlying in the right hand side of Figure 21. 

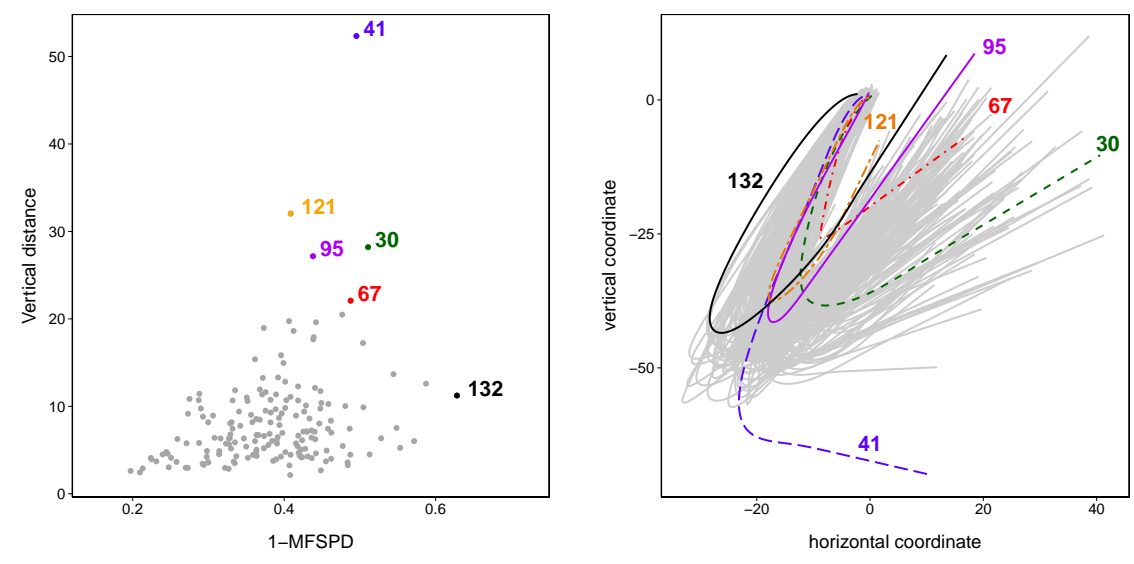

Fig. 20 Centrality-stability plot of the writing data. Fig. 21 Writing data with some outliers colored.
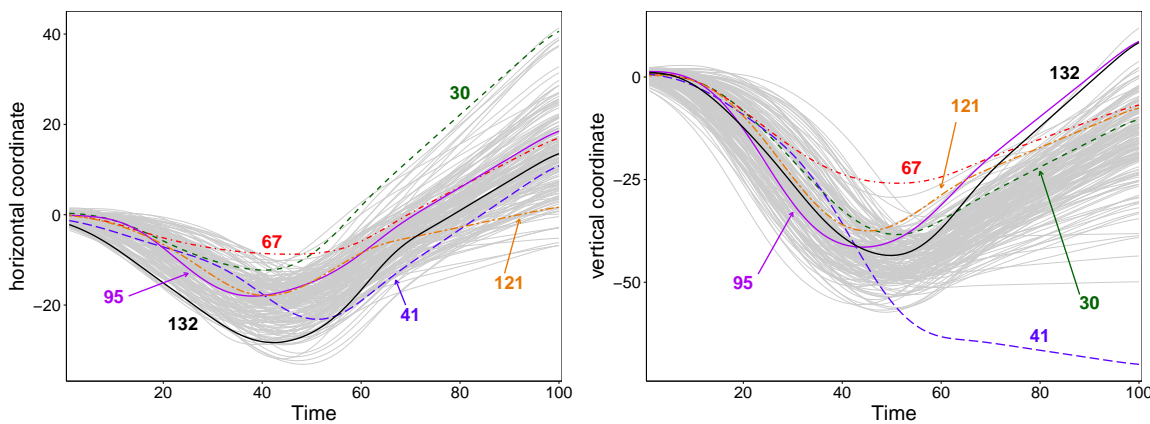

Fig. 22 Horizontal coordinate of the writing data Fig. 23 Vertical coordinate of the writing data with with some outliers colored. some outliers colored.

\subsection{Tablets Data}

As explained in Subsection 2.1 the tablets data consists of two groups of pills, and the data contains shift outliers and shape outliers. A common practice in chemometrics is to first apply a baseline correction, so from each curve we subtract its mean (over all wavenumbers). These baseline corrections are constant functions shown in Figure 24. The corrected spectra are displayed in Figure 25. From this plot it can already be seen that the lower curves (the shift outliers) are transformed to regular curves. If we do not want to lose the information that some of the curves were outlying prior to the preprocessing step, we can include the baseline functions in our analysis. Moreover, to increase the potential of finding shape outliers, we include the derivatives of the curves (shown in Figure 3) as well. All together we thus perform our analysis on a three-dimensional functional data set.

First we look at the SPD heatmap in Figure 26 and the AO heatmap in Figure 27. Both show the top 50 rows out of 90 . These heatmaps reveal interesting structures in the data. Curves 71 through 90 show an increased cross-sectional outlyingness between wavenumbers 8850 and 8950 . Among the rows with the highest functional AO 

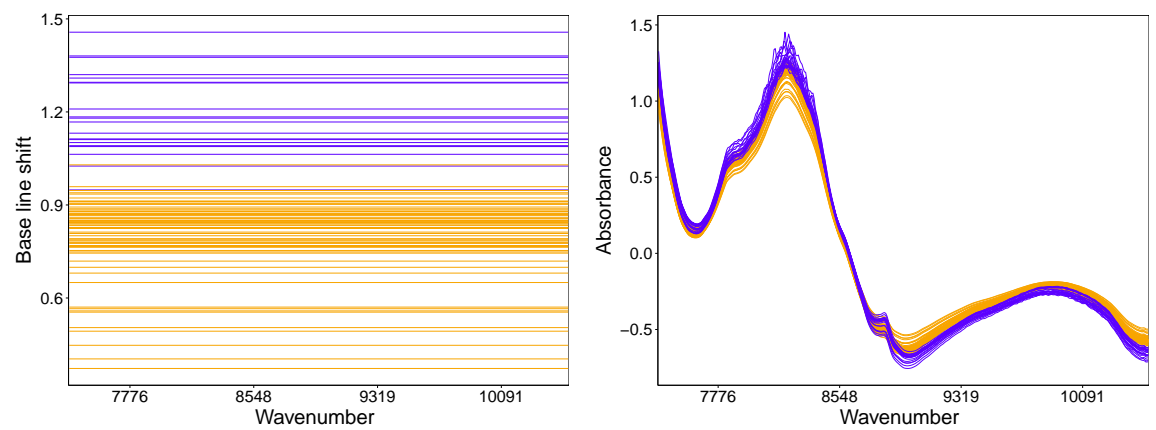

Fig. 24 Baseline correction functions of the tablets Fig. 25 The baseline-corrected spectra. data.

we also find curves 1 through 10. A similar pattern can be seen in the depth heatmap. However, there is a difference between these two groups. The cross-sectional adjusted outlyingness of the first 10 observations is mostly stable across wavenumbers, whereas the other outliers show more variability in their AO values.

This difference becomes more explicit in the centrality-stability plot (Figure 28). We clearly distinguish three groups of observations: regular ones, curves with low depth and stable outlyingness, and those with low depth and highly variable AO values. They correspond nicely to the three types of curves that could be seen on the raw data: the $90 \mathrm{mg}$ pills (orange), the downward shifted $90 \mathrm{mg}$ (red), and the 250 mg (purple), see Figure 29. As expected the shifted outliers have an almost constant deviation from the functional median and thus are situated in the lower right corner of the centrality-stability plot. The wiggly behavior of the $250 \mathrm{mg}$ tablets causes them to be located in the upper right part.

One might wonder whether these groups could also be found by applying existing univariate outlier detection tools to the marginal curves. We first apply the functional boxplot of Sun and Genton (2011) to each of the three univariate functional data sets. On the baseline functions (Figure 24), observations 1, 2, 4-6, 71-74, 79, 83, 88 and 90 are flagged as outliers. On the baseline-corrected spectra the functional boxplot indicates tablets $1,4,73,76,79,83$ and 90 as outliers. Applied to the derivatives it flags observations 1, 4, 5, 71-84 and 87-90. Even if we combine the results from the three components, tablets 3, 7-10, 85 and 86 are not detected.

Next we apply the outliergram which has recently been proposed by Arribas-Gil and Romo (2014). It combines two univariate functional depth methods: the modified band depth (MBD) and the modified epigraph index (MEI). Arribas-Gil and Romo (2014) show that for each curve

$$
\mathrm{MBD} \leqslant a_{0}+a_{1} \mathrm{MEI}+a_{2} n^{2} \mathrm{MEI}^{2}
$$

with $a_{0}, a_{1}, a_{2}$ depending on $n$ only. Using this relation they propose to plot the depths of the observations in the (MBD, MEI)-plane. They then define shape outliers as those observations whose distance $d_{i}=a_{0}+a_{1} \mathrm{MEI}_{i}+a_{2} n^{2} \mathrm{MEI}_{i}^{2}-\mathrm{MBD}$ is flagged as an outlier on the boxplot of the $d_{i}$. 


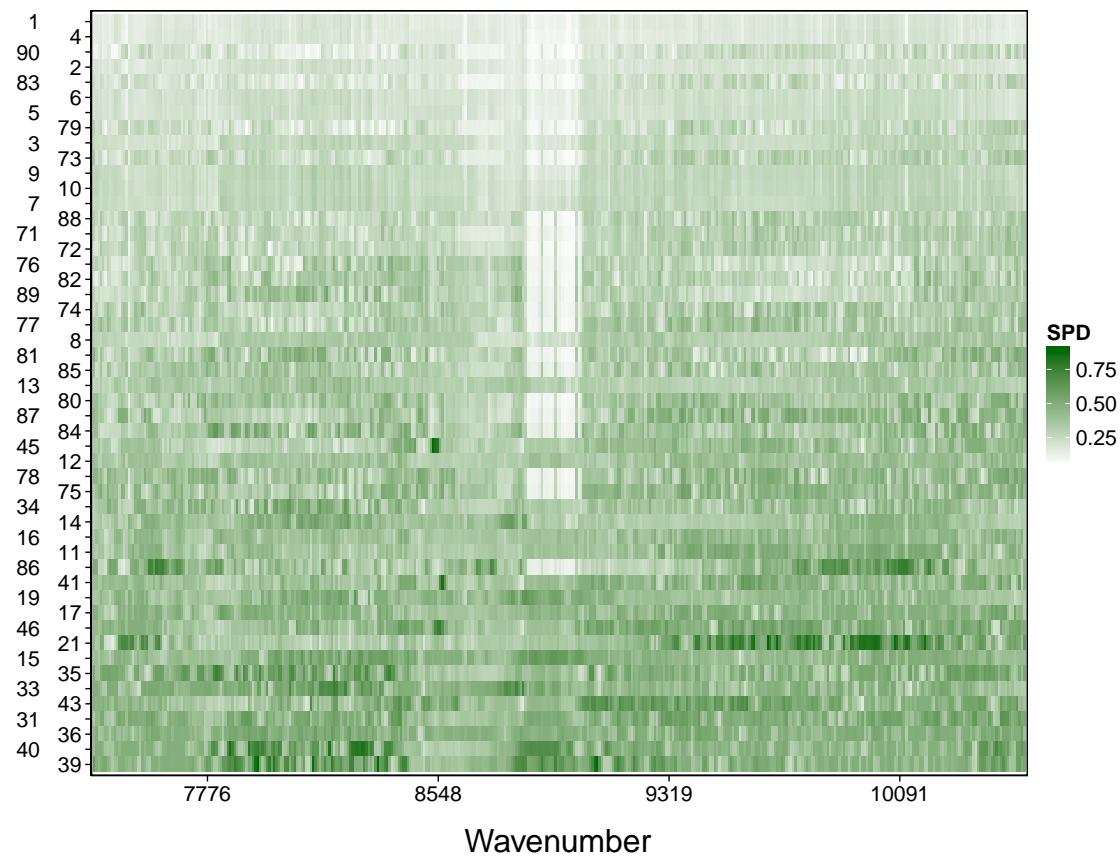

Fig. 26 SPD heatmap of the tablets data (top 50 rows).

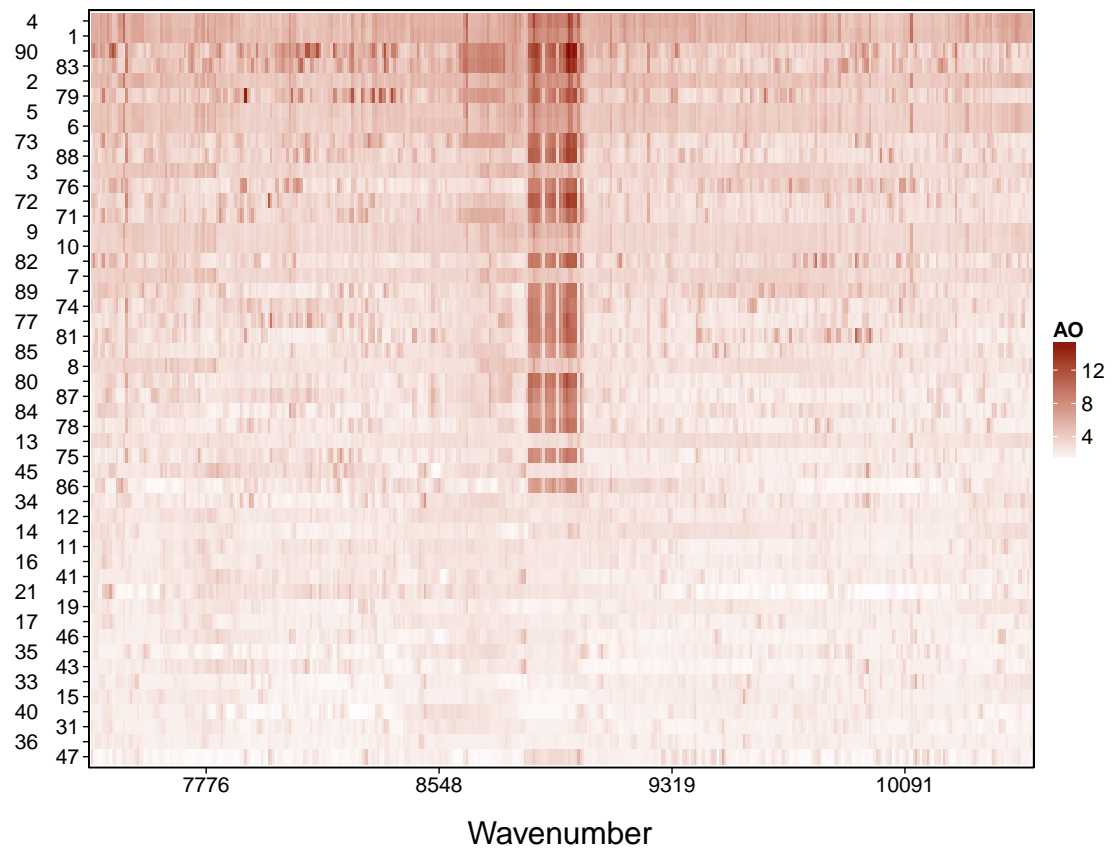

Fig. 27 AO heatmap of the tablets data (top 50 rows). 


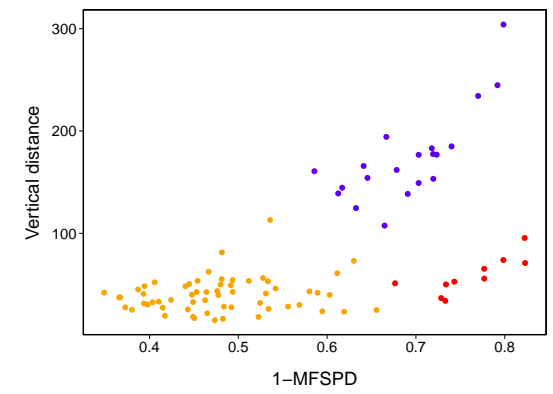

Fig. 28 Centrality-stability plot of the tablets data.

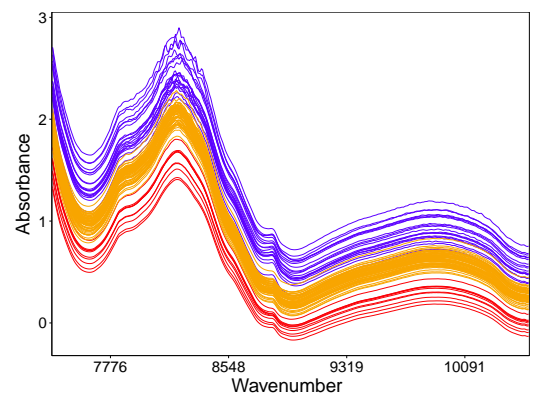

Fig. 29 Raw tablet curves colored according to their type.

If we apply this technique to the marginals of our three-dimensional functional data set, no outliers are detected from the baseline functions or the baseline-corrected spectra. Figure 30 shows the outliergram (right) of the derivatives of the spectra (left). Only observation 90 is flagged as a shape outlier and is marked on the left plot. Our multivariate analysis is thus much more powerful here.

\section{Conclusion and outlook}

We extend the toolbox of multivariate functional data analysis by new techniques for outlier detection. Functional data can have various kinds of outlyingness. A curve can have isolated or persistent outlying behavior, and the latter can be of the shift, amplitude, or shape variety, or a combination of those. The multivariate nature of the data makes these harder to detect than in the univariate functional case.
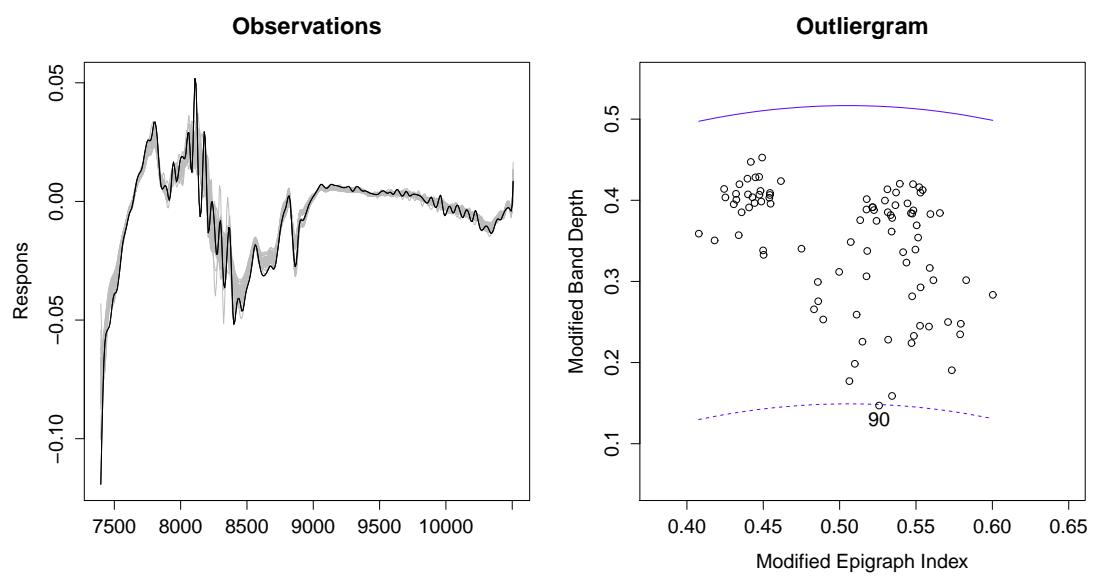

Fig. 30 Derivatives of the tablet spectra (left) and their outliergram (right). Curve 90 is shown in black in the left panel. 
Our new tools are based on depth-related notions so they do not have to assume any symmetry. We introduce the bagdistance, a metric based on halfspace depth. Even in the simpler case of multivariate nonfunctional data this is a new concept. The functional bagdistance carries more numerical information than the functional halfspace depth alone, and the new bagdistance heatmap helps to distinguish between isolated and persistent outlyingness.

A second approach is to extend the well-known projection depth to skewed distributions by basing it on the adjusted outlyingness (AO). In the multivariate case this skewness-adjusted projection depth (SPD) satisfies the depth axioms of Zuo and Serfling (2000a) and has convex depth regions. Integrating the SPD over time yields the functional SPD, as well as the SPD heatmap which can reveal detailed structure in the data. Combining information from the AO with the functional SPD we obtain a new diagnostic plot, which we call the centrality-stability plot because it contrasts the overall depth of a curve with the time-variability of its cross-sectional outlyingness. In the examples we illustrate the power of these multivariate functional tools, also compared to univariate analysis of the marginals.

We are currently working on a package for $\mathrm{R}$ and a corresponding toolbox for MATLAB implementing the techniques described in this paper. In future work we will investigate how these new tools can be used for other purposes such as supervised classification of multivariate data and of functional data.

\section{References}

Arribas-Gil A, Romo J (2014) Shape outlier detection and visualization for functional data: the outliergram. Biostatistics 15(4):603-619

Bache K, Lichman M (2013) UCI Machine Learning Repository. URL http://archive.ics.uci.edu/ml

Bai ZD, He X (1999) Asymptotic distributions of the maximal depth estimators for regression and multivariate location. The Annals of Statistics 27(5):1616-1637

Berrendero J, Justel A, Svarc M (2011) Principal components for multivariate functional data. Computational Statistics \& Data Analysis 55(9):2619-2634

Brys G, Hubert M, Struyf A (2004) A robust measure of skewness. Journal of Computational and Graphical Statistics 13:996-1017

Brys G, Hubert M, Rousseeuw PJ (2005) A robustification of Independent Component Analysis. Journal of Chemometrics 19:364-375

Claeskens G, Hubert M, Slaets L, Vakili K (2014) Multivariate functional halfspace depth. Journal of the American Statistical Association 109(505):411-423

Cuevas A, Febrero M, Fraiman R (2006) On the use of the bootstrap for estimating functions with functional data. Computational Statistics \& Data Analysis 51(2):1063-1074

Cuevas A, Febrero M, Fraiman R (2007) Robust estimation and classification for functional data via projection-based depth notions. Computational Statistics 22:481-496 
Dang X, Serfling R (2010) Nonparametric depth-based multivariate outlier identifiers, and masking robustness properties. Journal of Statistical Planning and Inference 140(1):198 - 213

Donoho D (1982) Breakdown properties of multivariate location estimators. $\mathrm{PhD}$ Qualifying paper, Dept Statistics, Harvard University, Boston

Donoho D, Gasko M (1992) Breakdown properties of location estimates based on halfspace depth and projected outlyingness. The Annals of Statistics 20(4):18031827

Dyrby M, Engelsen S, Nørgaard L, Bruhn M, Lundsberg-Nielsen L (2002) Chemometric quantization of the active substance in a pharmaceutical tablet using nearinfrared (NIR) transmittance and NIR FT-Raman spectra. Applied Spectroscopy 56(5):579-585

Esbensen K (2001) Multivariate Data Analysis in Practice, 5th edn. Camo Process AS

Febrero-Bande M, Galeano P, González-Manteiga W (2008) Outlier detection in functional data by depth measures, with application to identify abnormal $\mathrm{NO}_{x}$ levels. Environmetrics 19(4):331-345

Ferraty F, Vieu P (2006) Nonparametric Functional Data Analysis: Theory and Practice. Springer, New York

Fraiman R, Muniz G (2001) Trimmed means for functional data. Test 10:419-440

Hallin M, Paindaveine D, Šiman M (2010) Multivariate quantiles and multiple-output regression quantiles: from $L_{1}$ optimization to halfspace depth. The Annals of Statistics 38(2):635-669

He X, Wang G (1997) Convergence of depth contours for multivariate datasets. The Annals of Statistics 25(2):495-504

Hubert M, Vandervieren E (2008) An adjusted boxplot for skewed distributions. Computational Statistics and Data Analysis 52(12):5186-5201

Hubert M, Van der Veeken S (2008) Outlier detection for skewed data. Journal of Chemometrics 22:235-246

Hubert M, Van der Veeken S (2010) Robust classification for skewed data. Advances in Data Analysis and Classification 4:239-254

Hubert M, Claeskens G, De Ketelaere B, Vakili K (2012) A new depth-based approach for detecting outlying curves. In: Colubi A, Fokianos K, GonzalezRodriguez G, Kontoghiorghes E (eds) Proceedings of COMPSTAT 2012, pp 329340

Hyndman R (1996) Computing and graphing highest density regions. The American Statistician 50:120-126

Hyndman R, Shang H (2010) Rainbow plots, bagplots, and boxplots for functional data. Journal of Computational and Graphical Statistics 19(1):29-45

Ieva F, Paganoni AM (2013) Depth measures for multivariate functional data. Communications in Statistics - Theory and Methods 42(7):1265-1276

Larsen F, van den Berg F, Engelsen S (2006) An exploratory chemometric study of NMR spectra of table wines. Journal of Chemometrics 20(5):198-208

Liu R (1990) On a notion of data depth based on random simplices. The Annals of Statistics 18(1):405-414 
Liu X, Zuo Y (2014) Computing halfspace depth and regression depth. Communications in Statistics - Simulation and Computation 43(5):969-985

López-Pintado S, Romo J (2009) On the concept of depth for functional data. Journal of the American Statistical Association 104:718-734

López-Pintado S, Romo J (2011) A half-region depth for functional data. Computational Statistics \& Data Analysis 55:1679-1695

López-Pintado S, Sun Y, Lin J, Genton M (2014) Simplicial band depth for multivariate functional data. Advances in Data Analysis and Classification 8:321-338

Maronna R, Martin D, Yohai V (2006) Robust Statistics: Theory and Methods. Wiley, New York

Massé JC (2004) Asymptotics for the Tukey depth process, with an application to a multivariate trimmed mean. Bernoulli 10(3):397-419

Massé JC, Theodorescu R (1994) Halfplane trimming for bivariate distributions. Journal of Multivariate Analysis 48(2):188-202

Mizera I, Volauf M (2002) Continuity of halfspace depth contours and maximum depth estimators: diagnostics of depth-related methods. Journal of Multivariate Analysis 83(2):365-388

Mosler K (2013) Depth statistics. In: Becker C, Fried R, Kuhnt S (eds) Robustness and Complex Data Structures, Festschrift in Honour of Ursula Gather, Springer, Berlin, pp 17-34

Paindavaine D, Šiman M (2012) Computing multiple-output regression quantile regions. Computational Statistics \& Data Analysis 56:840-853

Pigoli D, Sangalli L (2012) Wavelets in functional data analysis: estimation of multidimensional curves and their derivatives. Computational Statistics \& Data Analysis 56(6):1482-1498

Ramsay J, Silverman BW (2002) Applied Functional Data Analysis. Springer Series in Statistics, Springer

Ramsay J, Silverman BW (2006) Functional Data Analysis, 2nd edn. Springer, New York

Ramsay JO, Li X (1998) Curve registration. Journal of the Royal Statistical Society, Series B 60(2):351-363

Romanazzi M (2001) Influence function of halfspace depth. Journal of Multivariate Analysis 77:138-161

Rousseeuw PJ, Leroy A (1987) Robust Regression and Outlier Detection. WileyInterscience, New York

Rousseeuw PJ, Ruts I (1996) Bivariate location depth. Applied Statistics 45:516-526

Rousseeuw PJ, Ruts I (1998) Constructing the bivariate Tukey median. Statistica Sinica 8:827-839

Rousseeuw PJ, Ruts I (1999) The depth function of a population distribution. Metrika 49:213-244

Rousseeuw PJ, Struyf A (1998) Computing location depth and regression depth in higher dimensions. Statistics and Computing 8:193-203

Rousseeuw PJ, Ruts I, Tukey J (1999) The bagplot: a bivariate boxplot. The American Statistician 53:382-387

Rousseeuw PJ, Debruyne M, Engelen S, Hubert M (2006) Robustness and outlier detection in chemometrics. Critical Reviews in Analytical Chemistry 36:221-242 
Ruts I, Rousseeuw PJ (1996) Computing depth contours of bivariate point clouds. Computational Statistics \& Data Analysis 23:153-168

Stahel W (1981) Robuste Schätzungen: infinitesimale Optimalität und Schätzungen von Kovarianzmatrizen. PhD thesis, ETH Zürich

Struyf A, Rousseeuw PJ (1999) Halfspace depth and regression depth characterize the empirical distribution. Journal of Multivariate Analysis 69(1):135-153

Struyf A, Rousseeuw PJ (2000) High-dimensional computation of the deepest location. Computational Statistics \& Data Analysis 34(4):415-426

Sun Y, Genton M (2011) Functional boxplots. Journal of Computational and Graphical Statistics 20(2):316-334

Tukey J (1977) Exploratory Data Analysis. Reading (Addison-Wesley), Massachusetts

Wang K, Gasser T (1997) Alignment of curves by dynamic time warping. The Annals of Statistics 25(3):1251-1276

Zuo Y (2003) Projection-based depth functions and associated medians. The Annals of Statistics 31(5):1460-1490

Zuo Y, Serfling R (2000a) General notions of statistical depth function. The Annals of Statistics 28:461-482

Zuo Y, Serfling R (2000b) On the performance of some robust nonparametric location measures relative to a general notion of multivariate symmetry. Journal of Statistical Planning and Inference 84:55-79

Zuo Y, Serfling R (2000c) Structural properties and convergence results for contours of sample statistical depth functions. The Annals of Statistics 28(2):483-499 